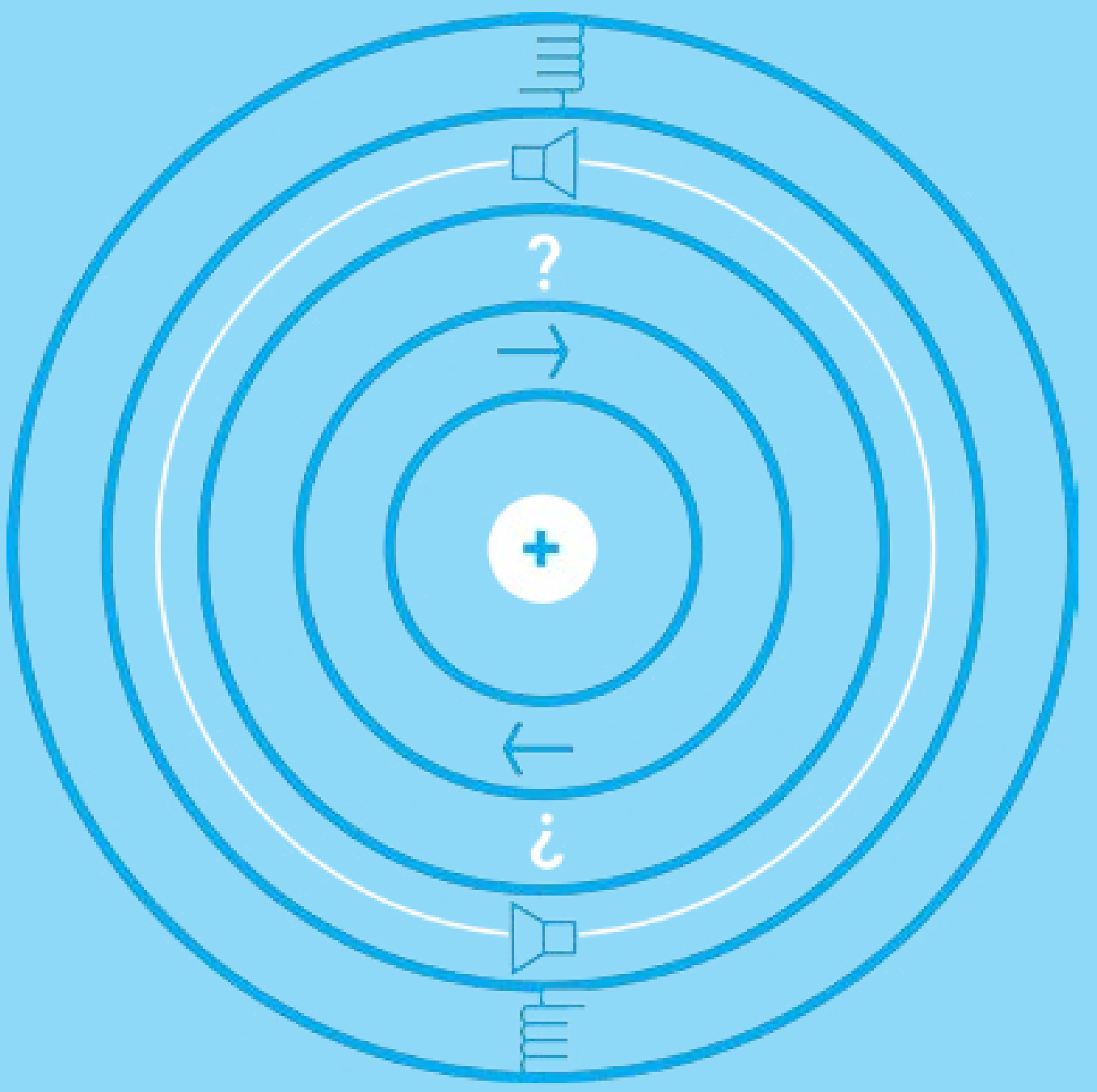

\title{
Maestros en Colectivo. Una década de actividad pedagógica
}

Teachers in group: A decade of pedagogical activity Professores em grupo: Uma década de atividade pedagógica Maestros en Colectivo 


\title{
Maestros en Colectivo ${ }^{1}$
}

\begin{abstract}
1 Maestros en Colectivo es un grupo de maestros investigadores de la escuela pública conformado desde hace más de una década e integrado por: James Frank Becerra Martínez, Colegio Simón Rodríguez; Especialista en Pedagogía de la Universidad Pedagógica Nacional. Correo electrónico: jafra8@ hotmail.com; Oscar Leonardo Cárdenas Forero, Colegio Entre Nubes; Magister en Desarrollo Educativo y Social de la Universidad Pedagógica Nacional-CINDE. Correo electrónico: osle1972@gmail.com; Sonia Milena Uribe Garzón, Colegio Entre Nubes; Magister en Desarrollo Educativo y Social de la Universidad Pedagógica Nacional-CINDE. Correo electrónico: sonia.smug80@gmail.com; María Anais Moncada Rodríguez, Colegio Gustavo Restrepo IED; Especialista en Multimedia para la Docencia, Universidad Cooperativa de Colombia. Correo electrónico: anais.moncada@gmail.com; Edith Constanza Negrete Soler, Colegio INEM Francisco de Paula Santander. Magíster en la Enseñanza de las Ciencias Exactas y Naturales de la Universidad Nacional de Colombia. Correo electrónico: negretesoler@gmail.com y Ángela Prieto Acuña; Ingeborg Cristina Behrentz Pfalz; Gildardo Moreno Cañadas y Juan José Garzón, interesados en las reflexiones pedagógicas alrededor de lo que se suscita en los Ambientes de Aprendizaje en el Aula (AAA).
\end{abstract}

Fecha de recepción: 24 de febrero de 2015 / Fecha de aprobación: 3 de abril de 2015.

\section{Resumen}

Este artículo de experiencia es una apuesta por describir el proceso de gestación y los retos y alcances logrados, en más de una década, por el equipo de investigación pedagógica Maestros en Colectivo, grupo constituido por docentes pertenecientes a la escuela pública y preocupado por explorar, reflexionar y proponer alternativas frente a lo que sucede en los Ambientes de Aprendizaje en el Aula. Su trabajo ha favorecido la producción de saber pedagógico, la transformación de las prácticas, la autoformación pedagógica, el encuentro discursivo, la cualificación del trabajo del maestro y la posibilidad de constituir nuevas formas de pensar al maestro y a la escuela en su totalidad.

\section{Palabras clave}

Maestros en Colectivo, Saber pedagógico, Seminario de formación permanente, autoformación, escuela.

\section{Summary}

This article of experience tries to describe the process of installation, the challenges and achievements made in more than a decade by a team of educational research Teachers Collective, group integrated by teachers from the public school that are concerned to explore, reflect and propose alternatives to what happens in Learning Environments in the Classroom; favoring the production of pedagogical knowledge, transforming practices, pedagogical self-education, the discursive meeting, the qualification of the teacher work and the possibility of creating new ways for thinking the teacher and the schools in its whole.

\section{Key words:}

Teachers collective, pedagogical knowledge, school, self-education and permanent education seminar.

\section{Resumo}

Este artigo e uma aposta para descrever o processo de gestação, os desafios e resultados alcançados feitos em mais de uma década por uma equipe de investigação educacional professores do collective. Grupo de professores da escola pública interessados em explorar, refletir e propor alternativas para o que acontece nos ambientes de aprendizagem em sala de aula, favorecendo a produção de conhecimento pedagógico, a transformação das práticas pedagógicas, auto-formação pedagógica, a reunião discursiva, a qualificação do trabalho docente e a possibilidade de criar novas formas de pensar o professor e as escolas em seu todo.

\section{Palavras chave:}

Professores do collective, conhecimento pedagógico, escola, autoformação e seminário de educação permanente. 


\section{A modo de presentación}

$\mathrm{E}^{\mathrm{n}}$ n el escenario contemporáneo, el discurso de la autoformación y cualificación de los sujetos se ha venido introduciendo con fuerza en todas las esferas de la sociedad; para lograr su propósito se ha considerando importante la creación de espacios llamados a favorecer las competencias y habilidades necesarias para facilitar el encuentro con el otro, el debate argumentativo, las concertaciones epistemológicas, las controversias $y$, en fin, la formación de subjetividades críticas, comunicativas y cognitivas que apuesten por la creación de conocimiento e innovación en los diferentes sectores de la sociedad.

Todo lo anterior dirige la mirada hacia la formación de grupos y colectivos de sujetos interesados por la autoformación y el desarrollo de las competencias para aprender a aprender. Así, propuestas de formación autónoma como la de "Maestros en Colectivo", instituidas en las fronteras de las escuelas, externas a las demandas de las políticas institucionales, cobran vigencia, validez e importancia, pues con el tiempo se consolidan como espacios: de encuentro pedagógico; de producción, transformación y validación del saber pedagógico, especialmente desde la reflexión sobre los Ambientes de Aprendizaje en el Aula (AAA); de proposición de alternativas didácticas y pedagógicas; y de creación, legitimación o invalidación de saberes escolares, pero además, como escenarios que posibilitan la profesionalización, cualificación y legitimación del quehacer del maestro.

Más allá de ir al pasado para encontrar el origen de los acontecimientos que justifican el presente, de rescatar la memoria para comprenderlo, o de excavar en el tiempo las líneas que permiten ver cómo un grupo de maestros se constituyó en lo que hoy se denomina "Maestros en Colectivo", la exploración que se pretende busca dar cuenta de lo que se es hoy: profesionales formados en el devenir entre la práctica y la reflexión, interesados en el acontecer de los AAA.

Maestros en Colectivo es entonces un equipo conformado por docentes que se desempeñan en diferentes instituciones educativas públicas, cuyos campos de acción abarcan desde la básica primaria hasta el nivel universitario; interesados por la investigación pedagógica, la producción y la cualificación de saber pedagógico y la participación y el encuentro con pares académicos y sociales para validar los conocimientos, comprometidos con unas prácticas de enseñanza y el quehacer en la escuela.
Los factores de autoformación desarrollados se han dado, tanto en los distintos colegios donde laboran los docentes participantes, que son fuente de observación y campo de acción, como en:

- La Corporación Escuela Pedagógica Experimental (CEPE), en donde se gestó y se hizo posible el encuentro preliminar del grupo.

- El Instituto para la Investigación Educativa y el Desarrollo Pedagógico (IDEP), que, a través de las distintas líneas de investigación, acción y participación, ha convocado y respaldado por años las propuestas de trabajo del colectivo, creyendo en que los maestros en Bogotá son productores de saber pedagógico.

- Las universidades, donde los maestros han obtenido una cualificación desde la institucionalidad.

- Las ONG y otras entidades que promueven los conversatorios, paneles, foros y ferias educativas.

- El Encuentro iberoamericano de colectivos y redes de profesores que hacen investigación en la escuela.

- Otras instituciones en las que se socializan y retroalimentan las experiencias y producciones pedagógicas.

Este es entonces, el preámbulo que fundamenta y orienta el acontecer de más de una década de actividad pedagógica, de Maestros en Colectivo.

\section{Una retrospección de la colectivi- dad "Maestros en Colectivo"}

Antes de la conformación del equipo, los docentes adelantaban individualmente, o desde el asilamiento de una práctica no institucionalizada:

[...] propuestas innovadoras que emergían de las inquietudes e intereses individuales y colectivos de los docentes frente a las situaciones del ambiente escolar [...] para el enriquecimiento de la práctica pedagógica y superar el solipsismo del maestro" (Becerra, et al, 2006, p. 15). 
Justamente, en el marco de la Expedición Pedagógica Nacional y bajo la tutela de la Corporación Escuela Pedagógica Experimental, acontecieron una serie de encuentros que llevaron a unos maestros a ubicar la pertinencia de conformar un equipo; inquietud que se fortaleció con una serie de talleres, conversatorios y con la socialización de las vivencias de los participantes en el aula, que adquiere sentido y se hace visible cuando las reflexiones se tornan en producciones (relatos, ponencias y relatorías), de esa manera se conforma el colectivo en torno a los AAA, definidos para esta colectividad como un:

[...] entramado de actores: sujetos reconocidos como seres integrales y diversos en gustos, intereses, valores, experiencias y formas de aprender y saber, actividad: correspondiente a las acciones y el hacer de los sujetos, a través de las cuales se vivencian experiencias y problemas de conocimientos (pedagógicos para el docente y escolares para los estudiantes) y contexto: condiciones sociales, políticas, históricas, culturales e ideológicas, ligadas un espacio-tiempo particular (Pedreros, 2006, p. 28).

Dicho entramado se configura y reconfigura gracias a las interacciones de los elementos que le conforman y que se mantienen en tensión, dotando a los AAA de un carácter dinámico. Para Maestros en Colectivo el acontecer de la clase es el marco de referencia para leer las distintas formas de concebir los AAA: "recogiendo vivencias propias y ajenas, tejidas por hilos invisibles entre los estudiantes y maestros, la actividad y el contexto [...] en tanto se reconoce el docente como constructor, en la reflexión de su práctica docente" (Becerra, et al, 2006, p. 30).

Con esto en mente, el trabajo del grupo llevó a la investigación, apoyada por el IDEP-COLCIENCIAS: "El Sentido de lo Público en el marco de la experiencia pedagógica Ambientes de Aprendizaje en el Aula: La auto-organización”, con la cual se reconoció que el trabajo colectivo posibilita la auto-organización y la construcción del sentido de lo público, asumido éste como el espacio de deliberación, de confrontación, diálogo, debate, negociación y acción, en el cual las personas toman decisiones relacionadas con la determinación de problemáticas e intereses comunes, por consiguiente: "[...] es necesario que la escuela, considere la posibilidad de dar apertura a nuevas formas de relacionarse, de reconocerse, de asumir, ver, estar y ser en el mundo, de convivir con los demás, de constituirse como sujeto, como comunidad, como cultura" (Maestros en Colectivo, 2009, p. 81).

\section{El Seminario de Formación Permanente: Espacio de encuentro pedagógico}

En la actualidad es constante la aparición de distintas prácticas, modos de actuación y de subjetivación, que instauran a los maestros en la sociedad de manera diferente e implican el surgimiento y la conjunción de diversas de formas comprensión de la escuela, la pedagogía y la educación. En este marco, se resalta el valor del encuentro de formación permanente, no solo como una instancia para reflexionar sobre las prácticas de enseñanza en la escuela, sino para que los docentes se constituyeran como sujetos productores de saber.

Uno de los intereses fundamentales establecido en el marco de los propósitos planteados en 1982 por el Movimiento Pedagógico, fue la formación de los maestros, que permitió concretar diversos espacios de formación alternativos a la escena institucional. Escenarios de cualificación de la labor del maestro, de construcción del saber pedagógico que posibilitaron la transformación del estatus del maestro, y de su formación, hacia un discurso que reconoce su práctica desde la perspectiva de que él ingresa a la escuela para transformar los modos de ser. Basados en estos principios, surge Maestros en Colectivo, un equipo que centra su interés en los AAA, con la intención de cualificar la labor docente.

Así, el Seminario de Formación Permanente es el pilar de Maestros en Colectivo, grupo que irrumpe para permitir que los docentes sospechen del acontecer que se instituye como verdad en los diversos contextos de la sociedad; ofreciendo la posibilidad de crear y validar los espacios pedagógicos, en los que desarrolla debates y modos de proceder frente a los asuntos de orden pedagógico, constituyendo subjetividades productoras de saber. Justamente en este espacio es factible la autoformación pedagógica, el encuentro discursivo, la cualificación y la posibilidad de constituir nuevas formas de pensar al maestro, a sus prácticas pedagógicas y a la escuela en su totalidad.

Es más, el Seminario de Formación Permanente se ha venido reafirmado y consolidado como un espacio de cualificación pedagógica de los docentes participantes del colectivo. En él se han puesto en juego, entre otros temas, los imaginarios, prácticas, experiencias, discursos y saberes que caracterizan las maneras particulares de proceder en el aula; se discuten asuntos 
epistemológicos, metodológicos y didácticos relacionados con la investigación, la innovación y el sentido de la educación y la pedagogía. Las producciones se han expuesto en espacios de socialización; pero, además, se han materializado en la creación documental y de alternativas para el aula y la escuela.

De este modo, el Seminario de Formación Permanente se asume como una posibilidad de encuentro y reconocimiento de los maestros y maestras que fortalece su imagen de sujetos constructores de saber pedagógico. Hecho que a su vez contribuye en el proceso, tanto de cualificación de su labor, como de consolidación de una colectividad dedicada a la formación, transformación y legitimación de los asuntos pedagógicos, mediante debates, discusiones, contrastes, validaciones y proyecciones que se ven reflejadas en la producción de saber, de documentos (ponencias, ensayos, artículos, informes de investigación, libros), así como en la ejecución de investigaciones, innovaciones y acciones pedagógicas a partir de la sistematización de experiencias $\mathrm{y}$ vivencias de conocimiento.

Las vivencias y exigencias del contexto contemporáneo llevan a que el maestro esté actualizándose permanentemente, en constante diálogo con el saber y sintiendo la imperiosa necesidad de sistematizar su trabajo y de participar en eventos académicos para tener una dimensión del estado de los procesos pedagógicos, metodológicos y didácticos; pero además, para socializar sus producciones, y sospechar de los hallazgos.

Esto significa que debe confrontar el conocimiento, el quehacer y la formación diaria para elaborar nuevos discursos sobre la acción pedagógica, que redunden en la re-significación de sus vivencias, emociones y pensamientos; en la generación de prácticas innovadoras, distintas a las institucionalizadas, y en la adquisición de otros imaginarios, para afrontar su desarrollo profesional y cualificar los procesos educativos. Así, el proceso de formación de los maestros es un aprender en el aprender que desarrolla su pensamiento crítico, la reflexión, el trabajo en colectivo y la investigación.

Como se observa, el Seminario de Formación Permanente poco a poco se distingue de otros espacios de encuentro y formación de los maestros, ya que se reconoce, en primera medida, como una instancia de cualificación y formación, pero también de autoformación; allí el colectivo destaca la importancia de la reflexión de la práctica pedagógica y de la proposición de alternativas que cambien los modos como se instalan ciertas prácticas pedagógicas en lo escolar.
Además, en el seminario se busca el reconocimiento del otro, entendiéndolo como una subjetividad con la que se construye el conocimiento pedagógico; se comparten vivencias escolares, experiencias, proyectos, necesidades, intenciones e intereses, ya que es precisamente con los colegas con quienes se valida (o invalida) el trabajo pedagógico. Con ellos, se generan nuevas construcciones en un espacio de continuo cuestionamiento, discusión y debate, de tal forma que cada uno se reconoce en un diálogo de saberes y en la interacción continua como sujetos de saber, de poder, de afectos y emociones.

La dinámica de trabajo ha llevado a que los maestros se auto-organicen y se auto-regulen, asumiendo roles, reconociendo potencialidades, apropiándose de compromisos, generando proyectos, definiendo tiempos de encuentro y buscando estrategias para elaborar escritos. Esta auto-organización también ha propiciado un proceso de regulación conjunta del devenir del colectivo, sus responsabilidades, dificultades, perspectivas y proyecciones, que se concretan en las maneras particulares de convivir, de establecer lazos de afectividad y de relacionarse. Esto ha permitido el reconocimiento del trabajo pedagógico adelantado por Maestros en Colectivo y su Seminario de Formación Permanente, no solo a nivel local, sino en algunos escenarios académicos nacionales e internacionales.

En la dinámica de Maestros en Colectivo, el Seminario de Formación Permanente adquiere un valor fundamental, pues permite el encuentro de maestros para debatir y deliberar sobre los diversos asuntos de los AAA. Con el paso de los años, el espacio ha asumido unas especificidades que lo vinculan con la idea de ser una instancia de cualificación y formación, ya que los docentes asumen que allí se posibilita su autoformación, pues llevan a cabo exploraciones epistemológicas, didácticas y pedagógicas que permiten cuestionar las prácticas de enseñanza y los discursos que sustentan estas actuaciones.

Todo ocurre en medio de un proceso continuo de enriquecimiento pedagógico; las vivencias y exigencias del contexto contemporáneo llevan a que el maestro se actualice, forme y desarrolle las competencias necesarias para un óptimo desempeño escolar. El trabajo le dirige a situaciones de constante diálogo, de producción académica e investigativa, de búsqueda teórica, de cuestionamiento constante, y a desarrollar propuestas pedagógicas que, a su vez, llevan a una participación periódica en eventos académicos para que le sea posible tener una dimensión sobre el estado de los paradigmas educativos y de los discursos que incursionan en la escuela para hacer de ella lo que es en 
cada momento; así progresa en la confrontación y elaboración de nuevos saberes y discursos sobre la acción pedagógica, que le permitan re-significar sus vivencias en el aula.

La dinámica del Seminario ha llevado a comprender la importancia del reconocimiento del otro, no solo como alguien que aporta y genera nuevas construcciones epistemológicas, didácticas o pedagógicas, que cuestiona y enriquece discursivamente, sino como un igual académico que convalida o interroga aquello que se instala como lo que se debe ejecutar en el aula. El otro se asume como alguien capaz de aprobar o refutar las prácticas pedagógicas de sus colegas dentro del colectivo, y esta es una actividad que enriquece las experiencias en el aula, de tal forma que cada uno se reconoce a sí mismo y al otro (colega del colectivo) como un sujeto de saber, con experiencias, un intelectual, con un ser afectivo y emocional que convive en una relación pedagógica basada en el respeto de las ideas.

El accionar del colectivo ha llevado a que se configuren nodos pedagógicos, es decir, ha creado líneas de investigación y de exploración pedagógica basadas en intereses o motivaciones desarrolladas al interior del colectivo; esto implica la constitución de subgrupos de estudio que se articulan a la intención general del equipo de maestros. Además, se perciben formas de auto-organización y auto-regulación cuando, por ejemplo, se definen los tiempos, la elección de los documentos a estudiar, la búsqueda de estrategias para elaborar escritos o la manera de acompañar a los colegas en la planeación de nuevas acciones o de adelantar las tareas para gestionar recursos e invitar a nuevos colegas.

Justamente, la proyección individual y/o colectiva aparece en el Seminario como espacio para compartir experiencias pedagógicas y ver sus posibilidades de ejecución en el aula; es un escenario para que se piense y repiense la escuela, se comprenda el discurso pedagógico y el sentido del educar. Pero también para explorar otros territorios de lo escolar y de lo educativo a fin de enriquecer las miradas sobre los AAA.

De tal manera, cada individuo, y el equipo en general, construyen y deconstruyen significados y sentidos, no solo de los AAA, sino de los discursos, modelos y paradigmas sobre la educación y la sociedad, de aquello que se instituye como natural y como axioma queda en la escuela, el pensamiento y las prácticas de los maestros. Así, la constitución de equipo de trabajo se convierte en una de las apuestas del grupo, pues el encuentro con el otro, el reconocimiento del saber pedagógico, la producción de escritos y el fortalecimiento de los lazos afectivos, ha permitido identificar inquietudes, generando empatías y el obje- tivo de organizar un equipo de trabajo alrededor de los intereses comunes.

En el Seminario de Formación Permanente se reconoce el valor de la participación, pues no solo es el valor por excelencia para compartir los puntos de vista, debatir las ideas y deliberar sobre los asuntos propuestos en cada sesión, sino que en ocasiones emerge como una tensión latente entre los maestros, pues da la oportunidad para pugnas asumidas desde los diferentes puntos de vista que están en juego.

Así, irrumpe la diversidad de argumentos que aprueban o se oponen a lo dicho por otros, develando los distintos marcos de diálogo de los docentes y posibilitado el reconocimiento de la importancia del respeto por la opinión del colega, la toma de decisiones a favor de argumentos convincentes y el establecimiento de acuerdos y negociaciones que no implican ceder la opinión propia, sino anteponer un saber en el que las voces de los maestros se ven reflejadas y reconocidas.

Conforme con lo dicho, el trabajo en el colectivo ha reconocido la existencia de diversas formas de organización de maestros (redes, grupos, colectivos, pares académicos) que les permite el reconocimiento de su quehacer pedagógico. Justamente, Maestros en Colectivo, en particular el Seminario de Formación Permanente, se asume como uno de esos espacios de encuentro docente en el que se aportan elementos para enriquecer las experiencias pedagógicas, que se traducen en la cualificación de las prácticas pedagógicas, en la transformación de los procesos de formación de los estudiantes y en la posibilidad de concebir el ambiente de aprendizaje de un modo distinto, uno en donde los maestros intenten superar dificultades como la insularidad y el aislamiento y favorecer procesos de formación, en ese encuentro con el otro, en una relación de pares académicos.

Por último, en la dinámica del Seminario de Formación Permanente, y como parte de su cultura organizativa, es necesario destacar que cada uno de los docentes participantes asume un rol, como el de moderador, encargado de otorgar la palabra a quien la solicita, o de relator, que lleva el acta de cada uno de los encuentros, la cual se lee y se somete a aprobación, después de los ajustes pertinentes, en la siguiente reunión; documento, que sirve como memoria pedagógica para la socialización de avances, el intercambio de experiencias en relación con los proyectos y vivencias de aula, y reconocimiento de los discursos de cada uno.

Así mismo, se abordan problemas relacionados con el planteamiento, retroalimentación y reajuste de propuestas de investigación o de producción escrita que se estén adelantando, 
en las cuales se reconoce y apoya al otro. Las dinámicas propias del Seminario han permitido la creación y adopción de nuevos roles y funciones, que surgen de acuerdo a las actividades y compromisos adquiridos, lo que deriva en la asignación de responsabilidades y de tareas en beneficio de la colectividad y del trabajo mismo, así como en la ejecución y registro de actividades específicas, individuales y conjuntas.

\section{Producción de saber}

- Participación en los Congresos Iberoamericanos de Educación realizados en Venezuela, Argentina y Perú (2008-2011, 2014).

- Encuentro de pedagogía, investigación y experiencias alternativas: Pedagogía para un mundo en crisis. Medellín, 2013.

\section{Publicaciones}

- La Escuela imaginada. Opciones de Autoformación. Red de Ciencias Naturales y Educación Ambiental. SEDCEPE. 2006.

- Maestros en Colectivo: Construyendo y reconstruyendo miradas y sentidos de los Ambientes de Aprendizaje. Bogotá, 2006.

- Ambientes de Aprendizaje en el Aula: Una experiencia en colectivo. IDEP, 2005.

- Los Ambientes de Aprendizaje en el Aula. Revista Nodos y Nudos, 2006.

- Reflexiones acerca de las relaciones escuela y contexto, 2005.

- El Sentido de lo público en el marco de la experiencia pedagógica Ambientes de Aprendizaje en el Aula: la Auto-organización, proyecto IDEP-Colciencias, 2007. 


\section{Referencias}

Becerra, J., et al. (2006, Julio-Diciembre). Maestros en colectivo: Construyendo y deconstruyendo miradas y sentidos de los Ambientes de Aprendizaje en el Aula. Nodos y Nudos, V. 3. No. 21. Bogotá: Universidad Pedagógica Nacional.

Becerra, J., et al. (2006). Los Ambientes de Aprendizaje en el Aula. La escuela imaginada. Opciones de autoformación. Bogotá: Magisterio.
Maestros en colectivo. (2009). La construcción del sentido de lo público en la escuela: La autoorganización. Universidad-escuela y la producción de conocimiento pedagógico. Bogotá: IDEP-Conciencias, Imprenta Distrital.

Pedreros, R., et al. (2006). Ambientes de Aprendizaje en el Aula: Una experiencia en Colectivo. Bogotá. 